\title{
Factores de riesgos asociados a la calidad de atención en pacientes sometidos a hemodiálisis
}

\author{
Dra. Lady Yuliette Bravo Loor \\ amy0082009@hotmail.com \\ https://orcid.org/0000-0002-3058-2203 \\ Hospital de Especialidades Portoviejo- Ecuador \\ Dra. Viviana Andrea Palacio Balda \\ vivi.palacio.1995@gmail.com \\ https://orcid.org/0000-0002-4304-2564 \\ Centro Medico San Gregorio Portoviejo- Ecuador \\ Dra. Karla Nohemy García Mejia \\ karlitamimi_taz8@hotmail.com \\ https://orcid.org/0000-0001-8295-2707 \\ Hospital de Especialidades Portoviejo- Ecuador \\ Dra. Arantxa Magdalena Chancay López \\ arantxa.chancay@gmail.com \\ https://orcid.org/0000-0002-1394-8752 \\ Hospital de Especialidades Portoviejo- Ecuador
}

\section{RESUMEN}

La enfermedad renal crónica y sus principales factores influyentes en la calidad de atención, se han convertido en una problemática de salud pública, en tanto que la prevalencia está representada por 2 personas por cada 100 habitantes. Los pacientes realizan una evaluación de sus condiciones de vida y de su enfermedad, generando la percepción de calidad de vida relacionada con la salud, evaluación que realiza el individuo respecto a su salud. El objetivo fue conocer los factores influyentes en la calidad de atención de los pacientes sometidos a hemodiálisis. El estudio fue, desde un enfoque empírico con un diseño cuantitativo de tipo analítico, de corte transversal prospectivo, mediante una revisión integrativa de búsqueda en las bases de datos Pubmed, Cuiden, Google académico, Search, Eric, Scielo y Meden. Resultados: De un total de 188 artículos preseleccionados, se excluyeron 96 durante la revisión de títulos y resúmenes. Otros 73 fueron eliminados tras la revisión del texto completo, se seleccionaron 19 artículos que cumplían con los criterios de inclusión/exclusión Conclusiones Los factores más influyentes en la satisfacción del paciente sometidos a hemodiálisis son, principalmente, la confianza transmitida y la disposición del personal de salud en la prestación de ayuda, el trato personalizado, la amabilidad y seguridad transmitida, la destreza para pinchar la fístula, el interés por la resolución de problemas asociados, así como la información relacionada con la higiene y la rapidez de respuesta requerida.

Palabras claves: estilos de vida; factores influyentes; insuficiencia renal; diálisis. 


\title{
Risk factors associated with the quality of care in patients undergoing hemodialysis
}

\begin{abstract}
Chronic kidney disease and its main factors influencing the quality of care have become a public health problem, while the prevalence is represented by 2 people per 100 inhabitants. The patients carry out an evaluation of their living conditions and their disease, generating the perception of quality of life related to health, an evaluation that the individual makes regarding their health. The objective was to know the factors influencing the quality of care of patients undergoing hemodialysis. The study was, from an empirical approach with a quantitative, analytical, prospective cross-sectional design, through an integrative search review in the Pubmed, Cuiden, Google academic, Search, Eric, Scielo and Meden databases. Results: Of a total of 188 pre-selected articles, 96 were excluded during the review of titles and abstracts. Another 73 were eliminated after reviewing the full text, 19 articles were selected that met the inclusion / exclusion criteria. Conclusions The most influential factors in patient satisfaction undergoing hemodialysis are, mainly, the confidence transmitted and the willingness of the staff to health in the provision of help, personalized treatment, kindness and security transmitted, the skill to puncture the fistula, interest in solving associated problems, as well as information related to hygiene and the speed of response required.
\end{abstract}

Key words: lifestyles; influencing factors; kidney failure; dialysis.

Artículo recibido: 10 Agosto. 2021 Aceptado para publicación: 07. Setiembre. 2021 Correspondencia: amy0082009@ hotmail.com Conflictos de Interés: Ninguna que declarar 


\section{INTRODUCCIÓN}

La enfermedad renal crónica (ERC) es consecuencia final de una serie de condiciones patológicas que acarrean a un daño renal definitivo, alterando las principales estructuras del riñón y conllevando a la pérdida progresiva de la función renal hasta ocasionar la muerte del paciente al no recibir una terapia de reemplazo renal oportuna., siendo un problema de salud pública. La Enfermedad Renal Crónica Avanzada (ERCA) es, hoy por hoy, un problema de salud pública en todo el mundo por su prevalencia e incidencia creciente en la población, su naturaleza crónica y permanente, su impacto en el paciente, su familia y el costo representativo para el sistema sanitario (David \& Sanjuán Hernández, 2018)

Los pacientes con ERCA, deben someterse a tratamientos no curativos, altamente invasivos, demandantes y que involucran altos costos para el paciente y su familia, a nivel físico, psicológico, social y económico. Entre los tratamientos de sustitución renal están el trasplante de riñón y la diálisis (peritoneal y hemodiálisis), los cuales deben acompañarse de una dieta estricta, toma de medicamentos y restricción de líquidos. La Enfermedad Renal Crónica Avanzada (ERCA) es, hoy por hoy, un problema de salud pública en todo el mundo por su prevalencia e incidencia creciente en la población, su naturaleza crónica y permanente, su impacto en el paciente, su familia y el sistema sanitario (Bermeo Minchala \& Jimenez Yora, 2014) Como consecuencia de esto, nos encontramos con una población cada vez más envejecida; lo que conlleva a un mayor número de problemas de salud y un aumento del uso de los recursos sanitarios.

Teniendo en cuenta la alta probabilidad de conductas negativas en el paciente renal sometido a hemodiálisis, se debe identificar a tiempo los diferentes factores que podrían estar alterando la adherencia terapéutica. Considerando que el gran porcentaje de pacientes con ERCA, experimentan etapas de crisis o negación durante el proceso de afrontamiento o asimilación de la enfermedad, ya que ellos jamás esperaron presentar esta patología y si a esto se suma que tienen que recibir un tratamiento sustitutivo (hemodiálisis), algo que ellos no conocen y que depende de ellos para sobrevivir, es normal la presencia de pensamiento de rechazo, esta a su vez es la conducta o comportamiento que toma para poder afrontar su enfermedad.

En el actual sistema salud, el ciudadano se ha convertido en centro y motor del mismo. Las necesidades del paciente son la base sobre la que se articulan las prestaciones 
asistenciales multidisciplinarias y la organización de los servicios de salud. El concepto de satisfacción se basa en la diferencia entre las expectativas del paciente y la percepción de los servicios que ha recibido; es decir, la percepción subjetiva, acompañada de la expectativa previa ( Sanz Turrado, Garrido Pérez , \& Caro Dominguez, 2017). Es un concepto complejo que está relacionado con una gran variedad de factores, como son el estilo de vida, las experiencias previas, las expectativas de futuro y los valores del individuo y de la sociedad.

Todo lo anterior puede tener serias implicaciones en la vida del paciente porque el hacer frente a una enfermedad crónica y progresiva, altamente demandante, cuyo tratamiento es invasivo y continuado, produce de manera permanente, importantes cambios en los estilos y hábitos de vida, entre ellos podemos mencionar ciertos factores condicionantes como la limitación en la dieta, los cambios en las relaciones sociales, familiares, laborales e incluso en el aspecto físico, están estrechamente relacionados con la aparición de trastornos emocionales en estos pacientes, lo que afecta seguramente la calidad de vida relacionada con la salud, aspecto importante que ha adquirido un interés relevante para ser estudiado, debido a la creciente incidencia y prevalencia de las enfermedades de este tipo.

La percepción de calidad de vida relacionada con la salud, se expresa en términos de bienestar, como un proceso dinámico y cambiante, que varía según el sistema de valores de los individuos ( Francoises Contrera, Esguerra, Espinosa, Gutiérrez, \& Fajardo, 2006). Hasta hace más de diez años, este concepto estuvo más centrado en las respuestas ante los efectos físicos, psicológicos y sociales que genera la enfermedad en la vida diaria del paciente y en la capacidad para recuperar el bienestar, no obstante, actualmente el concepto es visto más como una evaluación que realiza el individuo respecto a su salud, relacionada con el grado de funcionamiento social, físico y cognitivo, la movilidad y el cuidado personal para realizar las actividades cotidianas, así como el bienestar emocional y la percepción general de la salud.

Por lo anteriormente expuesto, la calidad de vida relacionada con la salud es un concepto en el que convergen múltiples factores, esta debe evaluarse a través de distintas áreas, abordando los dominios físico, psicológico y social. En el primero, se evalúan atributos como los síntomas, el funcionamiento físico y la discapacidad; en el dominio psicológico se consideran el nivel de satisfacción, el bienestar percibido, la 
ansiedad, la depresión y la autoestima; y en el tercer dominio se valoran las relaciones interpersonales del paciente, la actividad diaria y la rehabilitación laboral, entre otros. Esta valoración de la calidad de vida relacionada con la salud incluye aspectos objetivos y subjetivos, con el fin de realizar una evaluación completa e integral del individuo

\section{DESARROLLO}

Para los fines de esta investigación, se realizó un estudio desde un enfoque empírico de diseño cuantitativo de tipo analítico, de corte transversal prospectivo, que se centró en los factores influyentes de la calidad de vida de los pacientes sometidos a hemodiálisis, mediante una revisión integrativa de búsqueda en las bases de datos Pubmed, Cuiden, Google académico, Search, Eric, Scielo y Meden. Se incluyeron artículos científicos originales y de revisión, en inglés y español, excluyendo aquellos artículos que no presentaban resultados o que no estuviesen en texto completo.

Este tipo de revisión presenta un enfoque más sistemático y riguroso que la revisión narrativa de la bibliografía tradicional, estableciendo la consecución de criterios que aseguran el nivel de calidad deseado de los resultados obtenidos. El desarrollo de la revisión integrativa del presente estudio lleva consigo el establecimiento de diferentes fases bien definidas, destacando:

\section{Identificación del objetivo de la revisión}

¿Cuáles son los factores influyentes en la calidad de atención en pacientes sometidos a hemodiálisis?

\section{Búsqueda y selección de artículos}

La búsqueda de la bibliografía seleccionada se realizó a través diversas bases de datos con el objetivo de recopilar la mayor producción científica posible disponible en relación con el objeto a estudio. Como determinante, se estableció la selección de artículos publicados en los últimos 5 años (periodo comprendido entre 2016 y 2021). El periodo de búsqueda bibliográfica fue establecido desde junio al 31 de julio 2021.

El método de búsqueda consistió en utilizar como palabras clave o descriptores: Hemodiálisis (hemodialysis), satisfacción (satisfaction), paciente (patient), factores (factors). En cada base de datos se llevó a cabo estrategias de búsqueda diferentes, llevando consigo diversas pruebas de asociación entre los descriptores anteriormente citados mediante operadores boleanos (“AND”, “OR”), teniendo presente la existencia de cada uno de los descriptores empleados en: resumen, título del artículo y/o palabras clave. 
La metodología de búsqueda empleada fue determinada y condicionada a los siguientes criterios:

a) Criterios de Inclusión:

- Artículos en español e inglés.

- Artículos relacionados con el tema de interés.

- Artículos originales y de revisión a texto completo.

\section{b) Criterios de Exclusión:}

- Documentos provenientes de bases de datos sin evidencia científica ni fiabilidad derivada.

- Artículos que no presentasen los criterios de inclusión anteriormente mencionados.

\section{Lectura crítica}

Al inicio de la búsqueda no se consideró ningún criterio de inclusión/exclusión en las diversas bases de datos, con el objetivo de conocer la cantidad de producción científica existente respecto al tema a tratar, obteniendo, de este modo, mayor conocimiento del mismo, aceptándose todo aquel artículo que tratase sobre el tema en cuestión. Tras la lectura de títulos y resúmenes de todos los artículos potencialmente seleccionables, se descartaron aquellos que no cumpliesen con los criterios de inclusión y exclusión establecidos. Posteriormente, se realizó la lectura completa de los trabajos seleccionados, realizándose un nuevo cribado de aquellos que no versaban sobre el objeto a estudio.

Finalizado dicho proceso de selección, el número de artículos total que cumplían con los criterios de inclusión marcados y que se han utilizado para el estudio han sido de 19 (Tabla1). En ese momento se comenzó el análisis de los artículos recopilados.

Tabla 1 Procedencia de los artículos seleccionados.

\begin{tabular}{|l|c|c|c|c|c|c|}
\multicolumn{1}{|c|}{ Bases de Datos } & Pubmed & $\begin{array}{c}\text { Google } \\
\text { Académico }\end{array}$ & Search & Eric & Scielo & Meden \\
\hline $\begin{array}{l}\text { Total de publicaciones } \\
\text { localizadas }\end{array}$ & 35 & 57 & 22 & 36 & 32 & 6 \\
\hline $\begin{array}{l}\text { Total de publicaciones } \\
\text { seleccionadas }\end{array}$ & 4 & 5 & 3 & 1 & 4 & 2 \\
\hline
\end{tabular}

\section{Integración de los hallazgos}


La información analizada dentro de los artículos obtenidos fue: definición e identificación de factores influyentes en la calidad de atención en pacientes sometidos a hemodiálisis. Tras ello, se procedió a la interpretación de la información recopilada, así como el establecimiento de las conclusiones obtenidas.

\section{RESULTADOS}

De un total de 188 artículos preseleccionados, se excluyeron 96 durante la revisión de títulos y resúmenes. Otros 73 fueron eliminados tras la revisión del texto completo. Finalmente, se seleccionaron 19 artículos que cumplían con los criterios de inclusión/exclusión previamente establecidos, tal como se refleja en la (Figura 1).

Figura 1 Diagrama de flujo de las etapas metodológicas utilizadas en la Revisión Integrativa de la Literatura.

Características generales del estudio

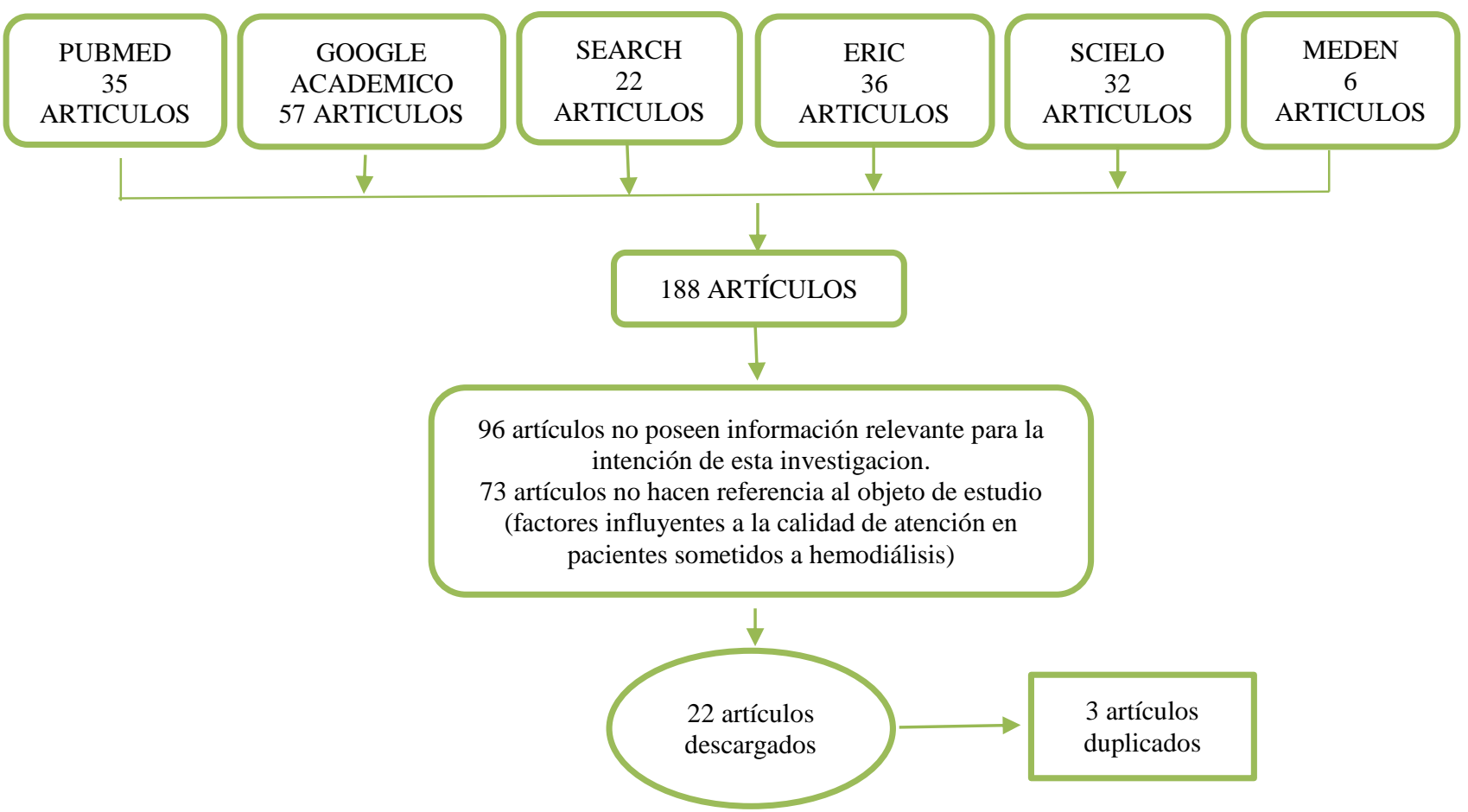

Los datos más relevantes de cada estudio se muestran en la Tabla 2.

\begin{tabular}{|c|c|c|c|c|}
\hline $\mathbf{N}^{\circ}$ & Autor, ano, País & $\begin{array}{c}\text { Diseño del } \\
\text { estudio }\end{array}$ & Muestra & Principales conclusiones \\
\hline 1 & 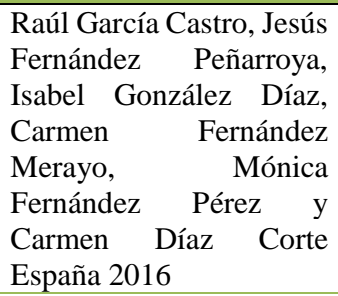 & $\begin{array}{l}\text { Estudios de } \\
\text { corte transversal }\end{array}$ & $\begin{array}{l}143 \\
\text { Participantes }\end{array}$ & $\begin{array}{l}\text { Un elevado porcentaje indica que los pacientes } \\
\text { colaboran de buen grado. Los pacientes en diálisis se } \\
\text { muestran satisfechos, pero debemos mejorar en la } \\
\text { información relacionada con la medicación. Este es el } \\
\text { punto que nos plantemos como objetivo de mejora }\end{array}$ \\
\hline
\end{tabular}




\begin{tabular}{|c|c|c|c|c|}
\hline 2 & $\begin{array}{l}\text { Sanz Turrado, } \\
\text { María; Garrido Pérez, } \\
\text { Luis; Caro Domínguez, } \\
\frac{\text { Carmen - España }}{2017 .}\end{array}$ & $\begin{array}{l}\text { Estudio } \\
\text { cualitativo de } \\
\text { tipo } \\
\text { fenomenológico, } \\
\text { mediante grupo } \\
\text { focal }\end{array}$ & $\begin{array}{c}7 \\
\text { Participantes }\end{array}$ & $\begin{array}{l}\text { Los pacientes en diálisis destacan la relación que se } \\
\text { crea entre ellos y enfermería. Tienen un alto grado de } \\
\text { confianza en el personal de enfermería, basado en su } \\
\text { destreza para pinchar la fístula arteriovenosa y en su } \\
\text { fiabilidad profesional. }\end{array}$ \\
\hline 3 & $\begin{array}{l}\text { Rodríguez González, } \\
\text { David; Sanjuán } \\
\text { Hernández, David - } \\
\text { España } 2018\end{array}$ & $\begin{array}{l}\text { Estudio } \\
\text { descriptivo y } \\
\text { correlacional }\end{array}$ & $\begin{array}{c}54 \\
\text { Participantes }\end{array}$ & $\begin{array}{l}\text { El índice de satisfacción fue de } 86,36 \% \text { para el ítem } \\
\text { referido a la satisfacción general, teniendo una } \\
\text { valoración de excelente. Los } 5 \text { ítems más valorados } \\
\text { fueron: la confianza trasmitida, la disposición del } \\
\text { personal, el trato personalizado, la amabilidad del } \\
\text { personal y la información relacionada con la higiene. } \\
\text { Los menos valorados fueron: la señalización para } \\
\text { llegar al Servicio, la capacitación del personal, la } \\
\text { información médica, la tecnología de los equipos y el } \\
\text { tiempo de espera para ser atendido por el médico }\end{array}$ \\
\hline 4 & $\begin{array}{l}\text { Cecilia María Díaz-Soto, } \\
\text { Paula Andrea Présiga- } \\
\text { Ríos, Carla María } \\
\text { Zapata-Rueda- } \\
\text { Colombia- } 2017\end{array}$ & $\begin{array}{l}\text { Estudio de tipo } \\
\text { analítico de } \\
\text { corte transversal } \\
\text { prospectivo }\end{array}$ & ------------- & $\begin{array}{l}\text { Los factores más influyentes en la satisfacción del } \\
\text { paciente en diálisis son, principalmente, la confianza } \\
\text { transmitida y la disposición del personal sanitario en } \\
\text { la prestación de ayuda, el trato personalizado, la } \\
\text { amabilidad y seguridad transmitida, la destreza para } \\
\text { pinchar la fístula, el interés por la resolución de } \\
\text { problemas asocia-dos, así como la información } \\
\text { relacionada con la higiene y la rapidez de respuesta } \\
\text { requerida }\end{array}$ \\
\hline 5 & $\begin{array}{l}\text { María Sanz Turrado, } \\
\text { Luis Garrido Pérez y } \\
\text { Carmen Caro } \\
\text { Domínguez- 2017- } \\
\text { Madrid }\end{array}$ & $\begin{array}{l}\text { Estudio } \\
\text { cualitativo, de } \\
\text { tipo } \\
\text { fenomenológico, }\end{array}$ & $\begin{array}{c}7 \\
\text { Participantes }\end{array}$ & $\begin{array}{l}\text { Este grupo de pacientes en hemodiálisis destaca la } \\
\text { relación que se crea entre ellos y enfermería. Tienen } \\
\text { un alto grado de confianza en el personal de } \\
\text { enfermería, basado en su destreza para pinchar la } \\
\text { fístula arteriovenosa y en su fiabilidad profesional. }\end{array}$ \\
\hline 6 & $\begin{array}{l}\text { Sor Fanny De Lourdes } \\
\text { Aguirre Bermeo- } \\
\text { Ecuador-2017 }\end{array}$ & $\begin{array}{l}\text { cuantitativo, } \\
\text { deductivo y de } \\
\text { nivel descriptivo } \\
\text { de corte } \\
\text { transversal }\end{array}$ & $\begin{array}{c}44 \\
\text { Participantes }\end{array}$ & $\begin{array}{l}\text { La intervención de enfermería es indispensables, ya } \\
\text { que a través de la educación, en alud el paciente logra } \\
\text { adquirir conocimientos, confianza y equilibrio } \\
\text { emocional para afrontar su situación de salud }\end{array}$ \\
\hline 7 & $\begin{array}{l}\text { Pupiales Guamán, } \\
\text { Angélica María- } \\
\text { Ecuador-2018 }\end{array}$ & $\begin{array}{l}\text { Estudio } \\
\text { diagnóstico, } \\
\text { descriptivo y } \\
\text { correlacional }\end{array}$ & $\begin{array}{c}30 \\
\text { Participantes }\end{array}$ & $\begin{array}{l}\text { La situación actual entre los factores psicosociales y } \\
\text { la calidad de vida, está determinada por la mayor } \\
\text { afectación hacia el sexo masculino, y al grupo de } \\
\text { personas adultas mayores y de la tercera edad, } \\
\text { mostrándonos una vez más la vulnerabilidad de esta } \\
\text { población, sin embargo no se puede echar de menos a } \\
\text { la población adulta joven que también se incluye } \\
\text { dentro de este contexto, afectando de alguna manera } \\
\text { a la productividad social }\end{array}$ \\
\hline 8 & $\begin{array}{l}\text { R. JOFRE- Madrid- } \\
2016\end{array}$ & $\begin{array}{l}\text { Estudio } \\
\text { descriptivo } \\
\text { empírico }\end{array}$ & -------- & $\begin{array}{l}\text { El objetivo debe de ser conseguir en cada paciente su } \\
\text { grado máximo de rehabilitación en función de sus } \\
\text { características. Es preciso implicar a otros } \\
\text { profesionales de la salud, como fisioterapeutas, } \\
\text { dietistas, etc., en el cuidado de nuestros pacientes. De } \\
\text { otra forma aspectos muy importantes pueden quedar } \\
\text { sin solucionar, y a pesar de los grandes avances } \\
\text { terapéuticos la insuficiencia renal seguirá teniendo un } \\
\text { impacto negativo en la calidad de vida }\end{array}$ \\
\hline 9 & $\begin{array}{l}\text { Dr. Gerardo Borroto } \\
\text { Díaz, Jacqueline } \\
\text { Almeida Hernández, Dr. } \\
\text { Amaury Lorenzo } \\
\frac{\text { Clemente - La Habana- }}{2017}\end{array}$ & $\begin{array}{l}\text { Estudio } \\
\text { prospectivo, } \\
\text { exploratorio y } \\
\text { descriptivo }\end{array}$ & $\begin{array}{c}52 \\
\text { Participantes }\end{array}$ & $\begin{array}{l}\text { Las mayores preocupaciones de estos enfermos se } \\
\text { centraban en el apoyo y las relaciones familiares, le } \\
\text { siguieron en importancia, la esfera sexual y la } \\
\text { reincorporación laboral. }\end{array}$ \\
\hline 10 & $\begin{array}{l}\text { Cecilia María Díaz-Soto, } \\
\text { Paula Andrea Présiga- } \\
\text { Ríos, Carla María } \\
\text { Zapata-Rueda }\end{array}$ & $\begin{array}{l}\text { Estudio desde } \\
\text { un enfoque } \\
\text { empírico de } \\
\text { diseño }\end{array}$ & $\begin{array}{c}51 \\
\text { Participantes }\end{array}$ & $\begin{array}{l}\text { Los programas de atención a pacientes con } \\
\text { enfermedad renal crónica que cuentan con equipos } \\
\text { interdisciplinarios que acompañan las diferentes }\end{array}$ \\
\hline
\end{tabular}




\begin{tabular}{|c|c|c|c|c|}
\hline & & $\begin{array}{l}\text { cuantitativo de } \\
\text { tipo analítico, de } \\
\text { corte transversal } \\
\text { prospectivo. }\end{array}$ & & $\begin{array}{l}\text { dimensiones del paciente, muestran un incremento en } \\
\text { la } \\
\text { calidad de vida relacionada con la salud y la } \\
\text { adherencia a los tratamientos farmacológicos. }\end{array}$ \\
\hline 11 & $\begin{array}{l}\text { Lic. Chipana Castro, } \\
\text { Karen Lic. Flores } \\
\text { Vásquez, Luis Santiago- } \\
2020 \text { Chile }\end{array}$ & $\begin{array}{l}\text { Estudio } \\
\text { cuantitativo, de } \\
\text { método } \\
\text { descriptivo y de } \\
\text { corte } \\
\text { transversal. }\end{array}$ & $\begin{array}{c}90 \\
\text { Participantes }\end{array}$ & $\begin{array}{l}\text { Los factores personales hacen referencia al conjunto } \\
\text { de aptitudes, actitudes, conocimiento y creencias que } \\
\text { el individuo puede poseer. Tanto el aspecto físico, } \\
\text { psicológico, como también, el estado de ánimo, } \\
\text { voluntad, el entorno cultural y social del paciente, el } \\
\text { nivel de educación, el conocimiento de la } \\
\text { enfermedad, la baja motivación, el estado cognitivo- } \\
\text { afectivo, el no percibir la necesidad del tratamiento; } \\
\text { el entender mal y no aceptar la enfermedad, así como } \\
\text { su personalidad, condicionan el resultado del } \\
\text { tratamiento y también, el de la adherencia terapéutica. }\end{array}$ \\
\hline 12 & $\begin{array}{l}\text { Yadira Pabón-Varela , } \\
\text { Kimberly Saray Paez- } \\
\text { Hernandez, Kelly } \\
\text { Dayanna Rodriguez- } \\
\text { Daza - Colombia } 2016\end{array}$ & $\begin{array}{l}\text { Estudio de } \\
\text { revisión } \\
\text { bibliográfica en } \\
\text { las bases de } \\
\text { datos }\end{array}$ & & $\begin{array}{l}\text { La calidad de vida de los pacientes con insuficiencia } \\
\text { renal crónica se ve afectada, por lo que están sujetos } \\
\text { a cambios en su estilo de vida que van de la mano con } \\
\text { el tratamiento sustitutivo renal y sus complicaciones, } \\
\text { además, presentan deterioro de la función física, } \\
\text { mental, emocional, laboral y espiritual. }\end{array}$ \\
\hline 13 & $\begin{array}{l}\text { Sheila Palau Osuna- } \\
\text { España } 2017\end{array}$ & $\begin{array}{l}\text { revisión } \\
\text { sistemática }\end{array}$ & ---------- & $\begin{array}{l}\text { Factores como la edad, el sexo, la etnia, educación, el } \\
\text { empleo, el estado mental de los pacientes, la } \\
\text { adaptabilidad, las creencias culturales y religiosas, la } \\
\text { comorbilidad, estatus socioeconómico, apoyo } \\
\text { familiar, apoyo social se consideran predictores } \\
\text { demográficos } 16 \text { independientes de la calidad de vida } \\
\text { que afectan a la percepción del paciente de su } \\
\text { enfermedad. }\end{array}$ \\
\hline 14 & $\begin{array}{l}\text { Engreed Mariela } \\
\text { Lozada- Ecuador } 2016\end{array}$ & $\begin{array}{l}\text { observacional, } \\
\text { analítico y } \\
\text { transversal }\end{array}$ & $\begin{array}{c}60 \\
\text { Participantes }\end{array}$ & $\begin{array}{l}\text { Se manifestó que los factores como sexo, edad, estado } \\
\text { civil, tiempo de hospitalización, nivel de instrucción, } \\
\text { situación laboral, influyen en la percepción del } \\
\text { paciente sobre la calidad de atención de enfermería y } \\
\text { su satisfacción, esto se debe a que cada paciente } \\
\text { percibe un fenómeno social desde su realidad y está } \\
\text { conformada por dichos factores }\end{array}$ \\
\hline 15 & $\begin{array}{l}\text { Francoise Contreras; } \\
\text { Gustavo Esguerra; Juan } \\
\text { Carlos Espinosa; } \\
\text { Carolina Gutiérrez; } \\
\text { Laura Fajardo- Bogotá } \\
2017\end{array}$ & $\begin{array}{l}\text { Diseño de } \\
\text { comparación }\end{array}$ & $\begin{array}{c}33 \\
\text { Participantes }\end{array}$ & $\begin{array}{l}\text { Estas diferencias, aunque no son estadísticamente } \\
\text { significativas solo indican una tendencia a que la } \\
\text { calidad de vida sea percibida de forma diferente por } \\
\text { hombres y mujeres, y por solteros y casados, aspecto } \\
\text { que debe ser estudiado con mayor profundidad, en } \\
\text { términos de convivencia y apoyo social percibido. }\end{array}$ \\
\hline 16 & $\begin{array}{l}\text { Luis Garrido Pérez } \\
\text { España } 2017\end{array}$ & $\begin{array}{l}\text { Estudio } \\
\text { cualitativo, de } \\
\text { tipo } \\
\text { fenomenológico }\end{array}$ & $\begin{array}{c}7 \\
\text { Participantes }\end{array}$ & $\begin{array}{l}\text { Las necesidades del paciente son la base sobre la que } \\
\text { se articulan las prestaciones asistenciales y la } \\
\text { organización de los servicios sanitarios }\end{array}$ \\
\hline 17 & $\begin{array}{l}\text { Andrea Bowen Bucheli , } \\
\text { Osvaldo Fosado Téllez } \\
\text { Ecuador } \\
2020\end{array}$ & $\begin{array}{l}\text { método } \\
\text { SERVQUAL } \\
\text { y la técnica de } \\
\text { cluster }\end{array}$ & $\begin{array}{c}3.063 \\
\text { Participantes }\end{array}$ & $\begin{array}{l}\text { Las brechas calculadas, de manera general, } \\
\text { alcanzaron valores negativos, a excepción de la } \\
\text { dimensión empatía que sobrepasa el valor de cero en } \\
\text { un pequeño margen, por lo que puede considerarse } \\
\text { que en estas dimensiones no se alcanzan las } \\
\text { expectativas con las que entró el paciente. }\end{array}$ \\
\hline 18 & $\begin{array}{l}\text { Gina Cynthia Pérez } \\
\text { Colombia } 2018\end{array}$ & $\begin{array}{l}\text { Estudio de } \\
\text { revisión }\end{array}$ & & $\begin{array}{l}\text { El bienestar social tiene que ver con las funciones y } \\
\text { las relaciones, el afecto y la intimidad, la apariencia, } \\
\text { el entretenimiento, el aislamiento, el trabajo, la } \\
\text { situación económica y el sufrimiento familiar. }\end{array}$ \\
\hline 19 & $\begin{array}{l}\text { Iris Alarcón } \\
\text { Daniela Farías } \\
\text { Silvia Barrios } \\
\text { Chile } 2020\end{array}$ & $\begin{array}{l}\text { Búsqueda } \\
\text { sistemática de } \\
\text { revisión } \\
\text { bibliografica }\end{array}$ & & $\begin{array}{l}\text { Los factores que influyen en la CV fueron: edad, sexo, } \\
\text { presencia de comorbilidad y depresión, nivel } \\
\text { educacional, deterioro cognitivo, funcionalidad } \\
\text { familiar, nivel de autonomía, cantidad de tiempo } \\
\text { sometido a la terapia, mejor comprensión de la } \\
\text { enfermedad, alto nivel de autocontrol, estilo de } \\
\text { enfrentamiento a la enfermedad y percepción de las } \\
\text { personas acerca del equipo de salud }\end{array}$ \\
\hline
\end{tabular}




\section{Descripción de los resultados en función de las variables}

\section{Factores influyentes en la calidad de atención en pacientes sometidos a hemodiálisis}

La mayoría de artículos seleccionados evidencian que los principales factores influyentes en la calidad de atención en pacientes sometidos a hemodiálisis son, principalmente, la confianza transmitida y la disposición del personal de salud en la prestación de ayuda, el trato personalizado, la amabilidad y seguridad transmitida, la destreza para pinchar la fístula, interés por resolución de problemas asociados, así como la información relacionada con la higiene y la rapidez de respuesta requerida por el paciente.

Por otro lado, los ítems peor valorados por los pacientes, según la bibliografía revisada, serían: señalización para llegar al servicio, capacitación del personal, información médica, tecnología de los equipos, tiempo de espera de atención médica, así como la información referente a la dieta y medicación prescrita, destacando éste último como el ítem en el que los pacientes reportan mayor insatisfacción. Asimismo, varios trabajos científicos, coinciden en que la falta de personal en las unidades de diálisis sería un factor determinante en el incremento de insatisfacción de los pacientes.

\section{Interacción con enfermería. Aspectos más valorados}

Según diversos estudios, resulta evidente el crucial papel desempeñado por el profesional enfermero en el cuidado, seguridad y recuperación del paciente, en especial en aquellas entidades patológicas de carácter crónico, conllevando consigo, en algunos casos, tratamientos sustitutorios o paliativos con un marcado carácter permanente, como es la hemodiálisis. En este sentido, las funciones inherentes de Enfermería llevadas a cabo en el paciente renal, como son el adecuado tratamiento, educación o apoyo psicológico, permiten alcanzar unos estándares de calidad en los cuidados adecuados que, siendo enfocados sobre el contexto de la satisfacción, actúan sobre cada uno de los factores determinantes del mismo, intentando desarrollar los más altos niveles de satisfacción. De ahí, que la mayoría de autores pongan énfasis en la necesidad de una adecuada educación sanitaria, por parte del profesional facultativo y enfermero, del paciente y familia respecto al proceso clínico y las características asociadas al mismo, que repercutirá directamente en el paciente.

\section{DISCUSIÓN}

La Enfermedad Renal Crónica Aguda, caracterizada por su evolución progresiva, lleva consigo un tratamiento prolongado y paliativo ante el fracaso renal objetivado, requiriendo al final de la última etapa de la enfermedad un trasplante renal o alguna de 
las modalidades terapéuticas que conforman la diálisis, la Hemodiálisis o la Diálisis Peritoneal, en las que los pacientes precisan de unas necesidades especiales que repercuten en su estado bio-psicosocial, siendo éstas principalmente determinadas por la propia evolución clínica y el manejo terapéutico llevado a cabo aplicado a los mismos.

Actualmente, los profesionales implicados en la atención del paciente renal tienen a su disposición un abanico de herramientas de valoración que permiten conocer, objetivamente, multitud de aspectos de interés relacionados con el propio proceso clínico y la esfera psicosocial de este tipo de pacientes. Atendiendo al nivel de calidad percibida por parte de los usuarios de diálisis ante los cuidados de enfermería, la satisfacción reportada en los artículos seleccionados, es elevada, coincidiendo con la mayor parte de producción científica disponible. Las acciones derivadas de la educación en salud llevada a cabo, así como el establecimiento de un adecuado tratamiento y apoyo psicológico ejercido durante el proceso de atención terapéutica por parte del profesional enfermero, reportan los mayores resultados positivos de satisfacción de este grupo de pacientes.

\section{Limitaciones del estudio}

Como principales limitaciones de nuestro estudio cabe destacar los pocos estudios localizados y la variabilidad existente en la metodología e instrumentos de medida utilizados para evaluar la satisfacción, y, por tanto, las diferentes dimensiones utilizadas en cada uno de ellos no permiten agrupar resultados. También en los artículos seleccionados, no existe homogeneidad respecto a la técnica de hemodiálisis en la que se ha evaluado la satisfacción.

\section{CONCLUSIONES}

- Los factores más influyentes en la satisfacción del paciente sometidos a hemodiálisis son, principalmente, la confianza transmitida y la disposición del personal de salud en la prestación de ayuda, el trato personalizado, la amabilidad y seguridad transmitida, la destreza para pinchar la fístula, el interés por la resolución de problemas asociados, así como la información relacionada con la higiene y la rapidez de respuesta requerida. Al contrario, los factores menos satisfactorios son la escasa capacitación del personal, la información médica, la tecnología de los equipos, el tiempo de espera de atención médica, así como la información referente a la dieta y medicación prescrita.

- En cuanto a los atributos que los pacientes en hemodiálisis perciben como más satisfactorios en su relación con enfermería, destacan la amabilidad, disposición e 
interés, preparación técnica profesional, trato personalizado, confianza y seguridad clínica.

- Finalmente, es importante indicar la necesidad de evaluar periódicamente el grado de satisfacción del paciente en hemodiálisis, para no solo responder a sus necesidades sino también a sus expectativas y detección de áreas de mejora asistenciales. Este hecho resulta fundamental en la conformación y diseño de planes de gestión y atención de salud, conllevando una adecuada actuación clínica, así como el establecimiento de la seguridad clínica pertinente, siguiendo los más altos estándares de calidad.

\section{REFERENCIAS}

Aguilar Chalacán, M. F., Vela Benalcazar, C., Recalde, J. L., \& Negrete, R. (2021). Mesenteritis esclerosante: diagnóstico y tratamiento. Scielo, 16. doi:10.30944/20117582.707

Akram, S., Pardi, D., Schaffner, J., \& Smyrk, T. (2007). Mesenteritis esclerosante: características clínicas, tratamiento y resultado en noventa y dos pacientes.

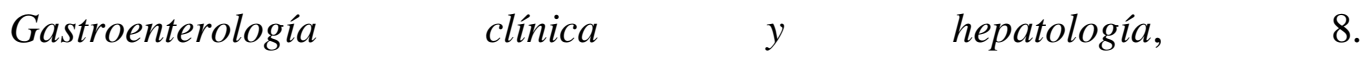
doi:https://doi.org/10.1016/j.cgh.2007.02.032

Aragón, E., \& Zapata, G. (01 de 2016). Evaluación de la calidad de vida en pacientes con enfermedad renal crónica en tratamiento sustitutivo. Cambios, 16. doi:https://doi.org/10.36015/cambios.v15.n1.2016.179

Emory , T., Monihan, J. M., \& Carr , N. (2017). Mesenteritis esclerosante, paniculitis mesentérica y lipodistrofia mesentérica: ¿una sola entidad? PubMed, 13. doi:10.1097 / 00000478-199704000-00004

Francoises Contrera, Esguerra, G., Espinosa, J. C., Gutiérrez, C., \& Fajardo, L. (2006). Calidad de vida y adhesión al tratamiento en pacientes con insuficiencia renal crónica en tratamiento de hemodiálisis. Universitas Psychologica. Recuperado el 04 de $06 \quad$ de 2021 de http://pepsic.bvsalud.org/scielo.php?script=sci_arttext\&pid=S165792672006000300005

Labalde Martíneza, M., Guijarro, J., Pacheco Martínez, P., Fernández Escuder, B., Domingo Asenjo, T., Jiménez de los Galanes, S., . . Perea Fernández, F. (2016). 
Mesenteritis esclerosante como causa excepcional de dolor abdominal. Elsevier, 12. doi:10.1016/j.gastrohep.2010.12.004

Lucas-Choez, M. M., Cevallos-Carrión, D. A., Quiroz-Figueroa, M. S., \& PiguaveFigueroa, T. J. (2021). Autocuidado y calidad de vida en pacientes renales con tratamiento de hemodiálisis. Polo del Conocimiento, 12. Recuperado el 01 de 08 de 2021, de file:///C:/Users/Usuario/Downloads/2292-12364-4-PB.pdf

Poniachik , J., \& Smok S, G. (2000). Mesenteritis retráctil Comunicación de cuatro casos. Scielo, 16. doi:http://dx.doi.org/10.4067/S0034-98872000001100010

Prado Solar, L. A., González Reguera, M., Paz Gómez, N., \& Romero Borges, K. (2014). La teoría Déficit de autocuidado: Dorothea Orem punto de partida para calidad en la atención.

Scielo,

6.

doi:http://scielo.sld.cu/scielo.php?script=sci_arttext\&pid=S1684-

$18242014000600004 \& \operatorname{lng}=$ es\&tlng=es.

Sanz Turrado, M., Garrido Pérez , L., \& Caro Dominguez, C. (2017). Factores que influyen en la satisfacción del paciente de diálisis con enfermería. Scielo, 20(1). $\begin{array}{lllllll}\text { Recuperado el de } & 08 \text { de }\end{array}$ https://scielo.isciii.es/scielo.php?script=sci_arttext\&pid=S225428842017000100009

Sharma, P., Yadav, S., Marie Needham, C., \& Feuerstadt, P. (02 de 2017). Mesenteritis esclerosante: una revisión sistemática de 192 casos. Springer, 10. doi:https://doi.org/10.1007/s12328-017-0716-5

Silva Tobar, S. D. (2016). HEMODIÁLISIS: ANTECEDENTES HISTÓRICOS, SU EPIDEMIOLOGÍA EN LATIONOAMÉRICA Y PERSPECTIVAS PARA EL ECUADOR. UNIANDES EPISTEME: Revista de Ciencia, Tecnología e Innovación., 3(1), 18.

Veletanga, J. (03 de 2016). Edicion medica. Recuperado el 04 de 08 de 2021, de https://www.edicionmedica.ec/secciones/salud-publica/en-ecuador-cerca-de-10mil-personas-necesitan-di-lisis-87408

Bermeo Minchala, M. L., \& Jimenez Yora, J. V. (2014). Evaluacion de la calidad de vida de los pacientes con Insuficicencia Renal Cronica sometidos a hemodialisis. Azoguez. Recuperado el 04 de 06 de 2021, de http://dspace.ucuenca.edu.ec/bitstream/123456789/21563/1/Tesis\%20Pregrado.p 
df

David, R. G., \& Sanjuán Hernández, D. (2018). Satisfacción percibida por los pacientes en el servicio de hemodiálisis del hospital general de La Palma. Redyalic, 15. $\begin{array}{lllllll}\text { Recuperado el de } & 2021 \quad \text { de }\end{array}$ https://www.redalyc.org/pdf/3598/359833150002.pdf

Delgado Plasencia , 1., Rodríguez Ballester , López Tomassetti , F., A Hernández Morales, A., \& Carrillo Pallarés . (2007). Paniculitis mesentérica: experiencia en nuestro centro. PubMed, 14. doi:10.4321 / s1130-01082007000500010

Navarro, S., \& Rivas Rivas, M. (2018). Mesenteritis esclerosante "aparente enfermedad maligna": alternativa diagnóstica ante la presencia de dolor, ascitis y suboclusión intestinal. Sociedad Andaluza de Patologia DIgestiva, 11. doi:This article is available from https://www.sapd.es/revista/2018/41/2/07

NMH Departamento de Nefrología de NMH. (Noviembre de 2019). Insuficiencia Renal: Elección del Tratamiento. Northwestern Medicine Hospital, 11. Recuperado el 05 de 08 de 2021, de file:///C:/Users/Usuario/Downloads/northwestern-medicineinsuficiencia-renal-eleccion-del-tratamiento-kidney-failure-treatments.pdf

OPS. (10 de 03 de 2015). Organizacion Panamericana de la Salud. Recuperado el 04 de 08 de 2021 ,

de https://www3.paho.org/hq/index.php?option=com_content\&view=article\&id=10 542:2015-opsoms-sociedad-latinoamericana-nefrologia-enfermedad-renalmejorar-tratamiento\&Itemid=1926\&lang=es

Organizacion Mundial de la Salud. (2020). COVID-19; Coronavirus; Ética en Investigación; Bioética. Organizacion Mundial de la Salud, Estados Unidos. Recuperado el 13 de 03 de 2021, de https://iris.paho.org/handle/10665.2/52142

Rodríguez Vidal, M., Merino Escobar, M., \& Castro Salas, M. (2009). VALORACIÓN PSICOMÉTRICA DE LOS COMPONENTES FÍSICOS (CSF) Y MENTALES (CSM) DEL SF-36 EN PACIENTES INSUFICIENTES RENALES CRÓNICOS EN TRATAMIENTO CON HEMODIÁLISIS. Ciencia y Enfermeria, 15. Recuperado el 05 de 08 de 2021, de https://www.redalyc.org/pdf/3704/370441798009.pdf

Sánchez, A., Albertini, C., Cárdenas, K., Pérez, G., \& Bécker, J. (2002). Mesentiritis esclerosante. Presentación de un caso y revisión de la literatura. Bibliografia 
Latinoamericana, 15.

Sociedad Española de Geriatría y Gerontología. (2006). Tratado de geriatria para residentes. En L. Z. Rubenstein. Madrid, España: Copyright. Recuperado el 04 de 08 de 2021, de https://www.segg.es/tratadogeriatria/pdf/s3505\%2000_primeras.pdf

Valladares Benitez, B. M., \& Bonilla Quevedo, G. L. (2017). "Supervivencia y desigualdad social en adultos mayores con Enfermedad Renal Crónica estadio 5 en tratamiento de hemodiálisis usuarios del centro CLINEF NORTE en el periodo comprendido de 01 enero de 2007 al 31 de diciembre de 2017". Quito. Recuperado el 05 de 08 de 2021, de http://repositorio.puce.edu.ec/bitstream/handle/22000/16727/SUPERVIVENCIA \%20Y\%20DESIGUALDAD\%20SOCIAL\%20EN\%20ADULTOS\%20MAYOR ES\%20EN\%20HEMODIALISIS.pdf?sequence=1\&isAllowed=y 